\title{
Comment on 'Wild-type APC prediction of poor prognosis in microsatellite-stable proximal colorectal cancer differs according to the age of onset'
}

\author{
José Perea ${ }^{\star}, 1,2,8$, María Arriba ${ }^{2,8}$, Daniel Rueda ${ }^{2,3}$, Ricardo Sánchez ${ }^{2}$, Juan Luis García ${ }^{4}$, Jessica Pérez ${ }^{4}$, Yolanda Rodríguez ${ }^{5}$,
} Rogelio González-Sarmiento ${ }^{4}$ and Miguel Urioste ${ }^{6,7}$

${ }^{1}$ Surgery Department, University Hospital 12 de Octubre, Madrid, Spain; ${ }^{2}$ Digestive Cancer Research Group, 12 de Octubre Research Institute, Madrid, Spain; ${ }^{3}$ Molecular Biology Laboratory, University Hospital 12 de Octubre, Madrid, Spain; ${ }^{4}$ Molecular Medicine Unit, Biomedical Research Institute of Salamanca (IBSAL), Institute of Molecular and Cellular Biology of Cancer (IBMCC), University of Salamanca-USAL-CSIC, Salamanca, Spain; ${ }^{5}$ Pathology Department, University Hospital 12 de Octubre, Madrid, Spain; ${ }^{6}$ Familial Cancer Clinical Unit, Spanish National Cancer Centre (CNIO), Madrid, Spain and ${ }^{7}$ Center for Biomedical Network Research on Rare Diseases (CIBERER), Institute of Health Carlos III, Madrid, Spain

Sir,

We read with interest the article by Jorissen et al (2015), highlighting as conclusion that $A P C$-wild-type (wt) status should be a marker of poor prognosis in microsatellite-stable (MSS) proximal colorectal cancer (CRC). Another important aspect underlined was that proximal APC-wt/MSS tumours exhibit features of the sessile serrated pathway, after testing that the prognostic value of the classification according to the APC status, and CRC location was reflected at the pathological and molecular level (Jorissen et al, 2015). They analysed 746 patients, and the median 'age of onset' of 70 years. Since a few years ago, we are trying to characterise an important subgroup within CRC, as it is early-onset CRC (EOCRC). To date, this subset of CRC appears to be different in comparison with others CRCs with older age of onset, especially those cases showing MSS (Boardman et al, 2013; Kirzin et al, 2014; Perea et al, 2014). We have shown this item, studying EOCRC from clinico-pathological, familial, and molecular points of view, and compared all the features found with a subset of late-onset CRC (LOCRC; diagnosed at an age of 70 years or older; Perea et al, 2014; Arriba et al, 2015).

Taking as the starting point the work by Jorissen et al (2015), we have analysed the same aspects, but applying an age of onset criterion, and compared a total of 56 cases of EOCRC with other 87 LOCRC cases. The methods used to analyze the microsatellite instability status, CpG islands methylator phenotype (CIMP), chromosomal instability by array comparative genomic hybridisation, as well as the clinico-pathological variables described, have been published before (Perea et al, 2014; Arriba et al, 2015). APC mutation status, as well as the other genes described by Jorissen et al (2015), have been analysed by next-generation sequencing.

First, the findings shown by Jorissen et al (2015) according to the prognostic results are confirmed only for LOCRC. In this particular age of onset, among patients with MSS tumours, APC-wt proximal cancers showed significantly inferior prognosis for overall survival (OS) and recurrence-free survival (RFS): 30 and 18 months, respectively, for proximal $A P C$-wt/MSS tumours compared with proximal APC-mutated (mt)/MSS tumours (OS: 56; RFS: 50), and distal-MSS tumours (both $\sim 40$ and 30 months, respectively). Even though, EOCRC subset showed interestingly different results. Worst prognosis subgroup in this age of onset was distal APC-mt MSS CRCs, with 55 months of OS and 40 months of RFS. In the progression in relation to a better prognosis, the other distal-MSS CRC group (APC-wt) appeared second, being those with the best prognosis, proximal APC-mt MSS cases (OS: 112; RFS: 96).

Jorissen et al (2015) tested as well that the prognostic value of the classification according to the APC status and CRC location was reflected at the pathological and molecular level: first, the poor prognosis $A P C$-wt/MSS cancers of the proximal colon consistently showed associations with features of the sessile serrated pathway, including poor differentiation, CIMP-high and BRAF mutation, and to a lesser extent mucinous histology and female gender. $A P C-\mathrm{mt} / \mathrm{MSS}$ distal cancers displayed the expected classic adenoma-carcinoma pathway features such as TP53 mutation and high IC, whereas $A P C-\mathrm{mt} / \mathrm{MSS}$ proximal cancers showed association with KRAS mutation and to a lesser extent with PIK3CA mutation, hallmarks of the alternate pathway. APC-wt/MSS distal cancers showed no consistently outstanding characteristics, although some tendency towards features of the sessile serrated pathway was noted. Although some of the subsets according to the APC mutation status and colon location are not too large in our series (proximal colon subsets), some conclusions should be given according to the differential clinico-pathological and molecular features showed by the APC status and colon location classification, when we compared them within the different age-of-onset subgroups. In relation to the EOCRC subgroup, the majority of the characteristics are equivalent for both proximal groups and the APC-mt/MSS distal cancers; only $A P C$-wt/MSS distal cancers differed, being an important group of sporadic cases without showing any predominant feature of all studied cases. On the other hand, LOCRC fulfilled mostly all the features described by Jorissen et al (2015), except with regard to the PIK3CA-mutation status.

These results not only continue suggesting the different behaviour, in this case in relation to the prognosis, of the MSS-EOCRC cases but also define more accurately one of the groups within the EOCRC whose molecular basis remains unknown, and therefore, for whom a greater effort in its search is necessary: APC-wt/MSS distal EOCRC.

\section{ACKNOWLEDGEMENTS}

This work was funded by projects PI13/01741 and PI13/0127 from the Spanish Ministry of Health and Consumer Affairs and FEDER, and was approved by the Ethics Committee of our Institution. We thank the Tumor Registry of the Pathology Department of the 12 de Octubre University Hospital for providing us with paraffin-embedded tissues.

\section{CONFLICT OF INTEREST}

The authors declare no conflict of interest.

\section{REFERENCES}

Arriba M, García JL, Inglada-Pérez L, Rueda D, Osorio I, Rodríguez Y, Álvaro E, Sánchez R, Fernández T, Pérez J, Hernández JM, Benítez J, GonzálezSarmiento R, Urioste M, Perea J (2015) DNA copy number profiling reveals different patterns of chromosomal instability within colorectal cancer according to the age of onset. Mol Carcinog; e-pub ahead of print 25 March 2015; doi:10.1002/mc.22315.

Boardman LA, Johnson RA, Viker KB, Hafner KA, Jenkins RB, Riegert-Johnson DL, Smyrk TC, Litzelman K, Seo S, Gangnon RE, Engelman CD, Rider DN, Vanderboom RJ, Thibodeau SN, Petersen GM, Skinner HG (2013) Correlation of chromosomal instability, telomere length and telomere maintenance in Microsatellite stable rectal cancer: A molecular subclass of rectal cancer. PLoS One 8(11): e80015.

Jorissen RN, Christie M, Mouradov D, Sakthianandeswaren A, Li S, Love C, Xu ZZ, Molloy PL, Jones IT, McLaughlin S, Ward RL, Hawkins NJ, Ruszkiewicz AR, Moore J, Burgess AW, Busam D, Zhao Q, Strausberg RL, Lipton L, Desai J, Gibbs P, Sieber OM (2015) Wild-type APC predicts poor prognosis in microsatellite-stable proximal colon cancer. Br J Cancer 113(6): 979-988.

Kirzin S, Marisa L, Guimbaud R, De Reynies A, Legrain M, Laurent-Puig P, Cordelier P, Pradère B, Bonnet D, Meggetto F, Portier G, Brousset P, Selves J (2014) Sporadic early-onset colorectal cancer is a specific subtype of cancer: a morphological, molecular and genetics study. PLoS One 9(8): e103159.

Perea J, Rueda D, Canal A, Rodríguez Y, ÁlvaroE, Osorio I, Alegre C, Rivera B, Martínez J, Benítez J, Urioste M (2014) Age at onset should be a major criterion for subclassification colorectal cancer. J Mol Diagn 16: 116-126. 